\title{
Generalized coupling in the Kuramoto model
}

Filatrella, G.; Pedersen, Niels Falsig; Wiesenfeld, K.

Published in:

Physical Review E

Link to article, DOI:

10.1103/PhysRevE.75.017201

Publication date:

2007

Document Version

Publisher's PDF, also known as Version of record

Link back to DTU Orbit

Citation (APA):

Filatrella, G., Pedersen, N. F., \& Wiesenfeld, K. (2007). Generalized coupling in the Kuramoto model. Physical Review E, 75(1), 017201. https://doi.org/10.1103/PhysRevE.75.017201

\section{General rights}

Copyright and moral rights for the publications made accessible in the public portal are retained by the authors and/or other copyright owners and it is a condition of accessing publications that users recognise and abide by the legal requirements associated with these rights.

- Users may download and print one copy of any publication from the public portal for the purpose of private study or research.

- You may not further distribute the material or use it for any profit-making activity or commercial gain

- You may freely distribute the URL identifying the publication in the public portal

If you believe that this document breaches copyright please contact us providing details, and we will remove access to the work immediately and investigate your claim. 


\title{
Generalized coupling in the Kuramoto model
}

\author{
G. Filatrella \\ CNR-INFM SuperMat Salerno and Department of Biological and Environmental Sciences, University of Sannio, Via Port'Arsa 11, \\ I-82100, Benevento, Italy \\ N. F. Pedersen \\ Oersted-DTU, Section of Electric Power Engineering, The Technical University of Denmark, DK-2800 Lyngby, Denmark \\ K. Wiesenfeld \\ School of Physics, Georgia Institute of Technology, Atlanta, Georgia 30332, USA \\ (Received 8 March 2006; revised manuscript received 22 September 2006; published 29 January 2007)
}

\begin{abstract}
We propose a modification of the Kuramoto model to account for the effective change in the coupling constant among the oscillators, as suggested by some experiments on Josephson junction, laser arrays, and mechanical systems, where the active elements are turned on one by one. The resulting model is analytically tractable and predicts that both first and second order phase transitions are possible, depending upon the value of a new parameter that tunes the coupling among the oscillators. Numerical simulations of the model are in accordance with the analytical estimates, and in qualitative agreement with the behavior of Josephson junctions coupled via a cavity.
\end{abstract}

DOI: 10.1103/PhysRevE.75.017201

PACS number(s): 05.45.Xt, 05.70.Fh, 74.81.Fa

Synchronization of coupled nonlinear oscillators appears almost ubiquitously in our world [1], to an extent that any listing will prove rather incomplete and unsatisfactory. The description of many nonlinear oscillators with different natural frequencies that must compromise to a common frequency requires a statistical treatment that is complicated by the nonlinear nature of the single elements. Most progress has been achieved within the framework of the theorist's playground for mutual entrainment, the Kuramoto model $[2,3](\mathrm{KM})$. However, the KM has a stringent characteristic: it assumes global coupling, with each oscillator having its own frequency $\omega_{j}$ coupled to all others on an equal footing through the sum of a periodic function and whose strength is tuned by a constant $K$

$$
\dot{\vartheta}_{i}=\omega_{i}+\frac{K}{N} \sum_{j=1}^{N} \sin \left(\vartheta_{j}-\vartheta_{i}-\alpha\right) .
$$

The intuitive idea behind the model is that each oscillator if uncoupled would oscillate at the frequency $\omega_{i}$, but the resulting phase difference with the others produces a restoring "force" (proportional to the coupling $K$ ), thus favoring synchronous motion. Usually one gladly pays the price of this simplification (global coupling) to be compensated by the analytical insight offered by the model. But sometimes the situation is better - for example when the cross talk between oscillators is mediated by a common connection to passive elements, say a linear resonator - since it is possible that the global coupling approximation is not merely a crude truncation of the coupling to some averaged mean field. For example, Josephson Junctions (JJ) coupled through a lumped resonator are described by the following equations (in normalized units):

$$
\ddot{\varphi}_{i}+\frac{1}{\sqrt{\beta}} \dot{\varphi}_{i}+I_{i} \sin \varphi_{i}=B-\dot{q},
$$

$$
\ddot{q}+\frac{1}{Q} \dot{q}+\Omega^{2} q=\frac{1}{\beta_{L}} \sum_{j=1}^{N} \dot{\varphi}_{j} .
$$

Here $\beta$ is the McCumber parameter, $B$ the normalized current through the junctions, $q$ the normalized charge of the RLC circuit of peak frequency $\Omega$ and quality factor $Q, \beta_{L}$ the coupling of the array to the RLC circuit. The oscillators' frequencies are determined by the normalized critical currents $I_{i}$ taken to be a Lorentzian distributed random variable of average $l$ and $\gamma$ width, $g(I)=(\gamma / \pi) /\left[\gamma^{2}+(\mathrm{I}-1)^{2}\right]$. In the limit of weak coupling to the resonator Eqs. (2) reduce to the KM (1) for the overdamped [4] and underdamped [5] cases. Simulations of (2) can show hysteresis (sometimes rather large hysteresis) while the Kuramoto model does not. The reductions in $[4,5]$ effectively ignore the dynamical nature of the coupling-degrees-of-freedom (i.e., the load), which is (we think) the reason for the hysteresis. Our remedy is to restore the dynamical-feedback aspect of the coupling while still retaining the essential phase-model reduction of $\mathrm{KM}$. One virtue of our approach is that it retains some generality, precisely because the model doesn't include "auxiliary" variables describing the coupler dynamics. A second virtue is that our modification is highly tractable. This connection offers hope that the Kuramoto paradigm might be directly and quantitatively tested in real Josephson arrays with resonator-coupling architecture, whose performance is known to depend on the degree of synchronization [6-9] (in a previous work [10] we have already explored this possibil-

\footnotetext{
${ }^{1}$ Ref. [5] quotes results for the general RLC load case, but it presents an explicit derivation only for the purely capacitative load, in which limit our Eqs. (2b) reduces to $\beta_{L} \Omega^{2}=\Sigma_{j} \dot{\varphi}_{j}$. Moreover, Ref. [5] employed a perturbation expansion in powers of the ratio of critical current to bias current; with our present choice of scaling, this ratio is just $I_{i} / B$.
} 
ity, that here will be discussed on a more general ground). In this paper we will therefore employ model (2) as an example of globally coupled rotators that might be described by KM type models. We emphasize that there are also other experiments where the number of active elements can be systematically tuned, such as laser arrays [11] and a recent recreation of London's Millennium Bridge instability [12,13]. In particular although existing experiments have not tried to determine this aspect of the dynamics, the Millennium Bridge measurements are consistent with possible hysteretic behavior of the transition to synchronous motion [14], which would be a clear indication of a first order transition. The key difference is the way in which the experimental systems are tuned across the transition. In the theoretical model (1) the natural control parameter is the coupling coefficient $K$, with $N$ held fixed. In contrast, the experiments in Refs. $[8,9,12,13]$ systematically increased the number of (active) oscillators, so one should keep $K$ constant in Eq. (1) and consider a finite $N$, see Ref. [15]. However the thermodynamic limit is very convenient for an analytical treatment, in fact in [15] it was possible to analytically treat the model only far from the transition coupling constant $K_{c}$ and for a compact distribution of the frequencies $\omega_{j}$. To circumvent this restriction we suggest another approach: to consider a very large (effectively infinite) number of oscillators and to assume that the effect of the increased number of active oscillators is to increase the coupling constant $K$ which embodies the physical consequences of this sequential activation of the oscillators. Our modified model is analytically tractable, and leads to some interesting predictions. Among these is the possibility of a first order transition at the onset of synchronization, a feature reported in the above-cited experiments. [In contrast, Eq. (1) leads to a second order transition if the frequency distribution density is unimodal.] In fact the main feature observed in experiments of underdamped $\mathrm{JJ}[8,9]$ is the existence of a threshold for synchronization, with sudden microwave emission much above the incoherent emission from unsynchronized arrays, similar to the sudden jump to a finite amplitude of the oscillations when the number of walkers exceeds a threshold in the Millennium Bridge recreation $[12,13]$. This signals a sudden increase in the degree of coherence, which is measured in the framework of the KM by the order parameter $r$

$$
r e^{i \psi}=\frac{1}{N} \sum_{j=1}^{N} e^{i \vartheta_{j}}
$$

that (roughly speaking) describes the fraction of locked oscillators (an incoherent state results in $r=0$, while a perfectly coherent state implies $r=1$ ). The Kuramoto analysis predicts that increasing the strength of the coupling from zero, has no effect on the order parameter up to a critical value of the interaction $K_{c}=2 /[\pi g(0)]$ [for $\alpha=0$ in Eq. (1) and $g$ being a unimodal and symmetric distribution density of the natural frequencies $\omega_{j}$ ]. Above this value $r$ increases monotonically toward the perfect synchronous state $r=1$, so that it is natural to describe the transition as a second order phase transition. However, simulations of system (2), adding the active oscillators one by one (and removing them in the same order),

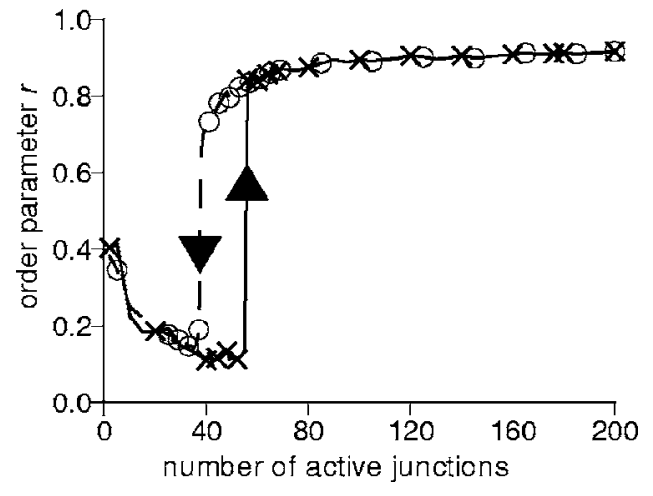

FIG. 1. Behavior of the order parameter for a Josephson Junction array coupled to a cavity obtained by increasing (crosses, solid line) and decreasing (circles, dashed line) the number of active junctions. The arrows denote the direction of the transition. Parameters of the simulations are: $B=0.6, \beta=10, \beta_{L}=5000, Q=100$, $\Omega=\left\langle\omega_{i}\right\rangle$. The disorder is set to $\gamma=0.05$.

reveal a different behavior of the order parameter $r$ (see Fig. 1). The sudden jump in $r$ at onset is just the feature seen in the experiments $[8,16]$. Since underdamped JJ are also described by a massless KM [5], this difference cannot be attributed to Kuramoto models with inertia, that lead to a first order phase transition [17]. We therefore conclude that the addition of oscillators one by one is the main cause of the sudden jump to a finite value of the order parameter $r$. The physical consequence of adding active junctions is to vary the amplitude of the resonator oscillations [see Eq. (2)], we therefore are led to a phenomenological variation of the KM that accounts for the change of the coupling with the number of active oscillators, i.e., we propose to model the number of active oscillators via the constant $K$ while keeping $N$ very large [effectively infinite, while in Eq. (2) $N$ is actually increased]

$$
\dot{\vartheta}_{i}=\omega_{i}+\frac{K(r)}{N} \sum_{j=1}^{N} \sin \left(\vartheta_{j}-\vartheta_{i}\right)
$$

Also here the oscillators' frequencies are taken to be a Lorentzian distributed random variable with zero average and $\gamma$ width, $g(\omega)=(\gamma / \pi) /\left(\gamma^{2}+\omega^{2}\right)$.

A simple functional form for $K(r)$ to describe this effect is the power-law $K(r)=K r^{z-1}$. In this general framework the $\mathrm{KM}$ is the special case of constant coupling $z=1$, for $z>1$ one gets models in which the coupling is enforced when more rotators are synchronized (see also Ref. [10] which considered the case of a linear dependence of the coupling on the order parameter, $z=2$, in connection with vortex motion in long JJ), while for $z<1$ the coupling is weakened. We do not have a derivation of the functional behavior of $K(r)$ as in Refs. [4,5,18]; therefore we will use the parameter $z$ as a heuristic measure of the strength of the feedback mechanism, which might also account for the shift of the average frequencies $\left\langle\omega_{i}\right\rangle$ with respect to the peak $\Omega$ of the resonator. Simulations of the Eqs. (4) for various values of the exponent $z$ are reported in Fig. 2, together with the analytic predictions to be derived below. For the sake of comparison, we 

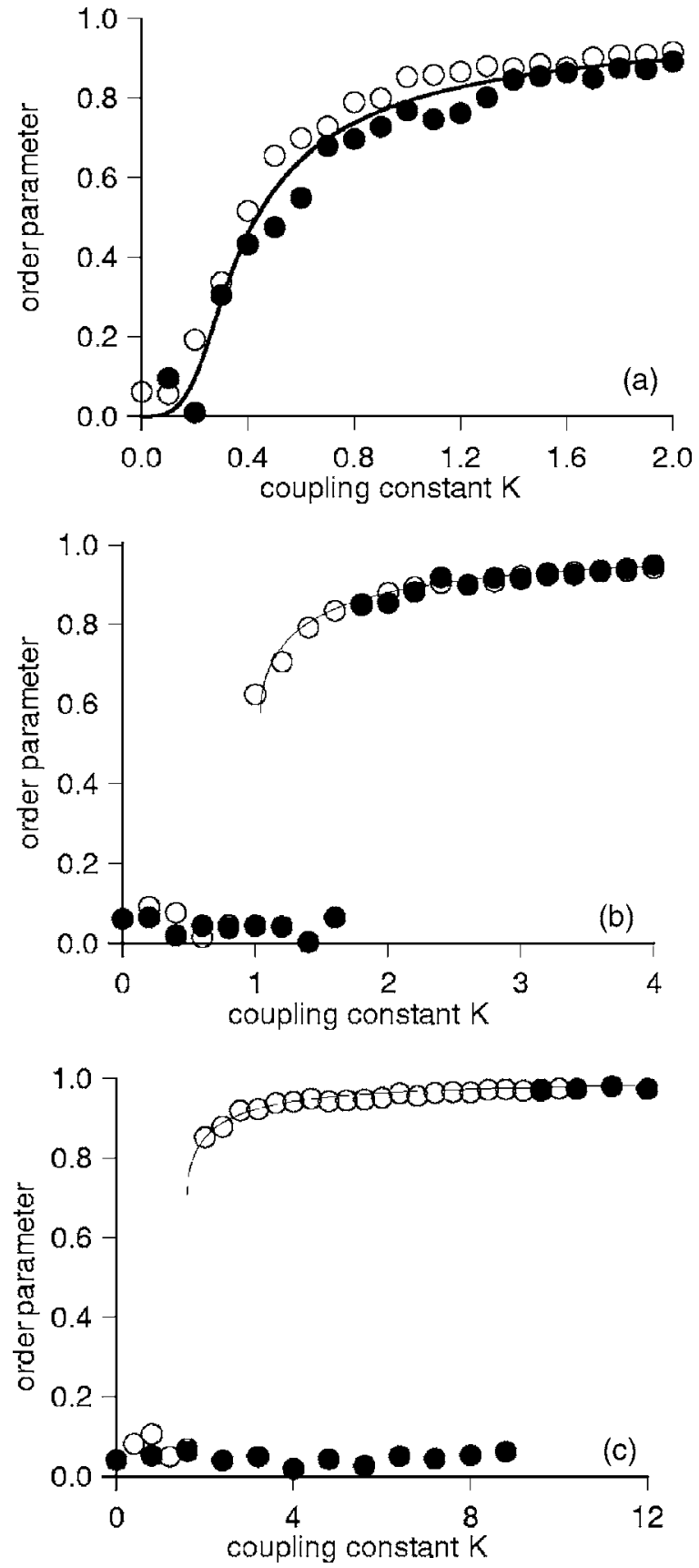

FIG. 2. Numerical and analytical results of Eq. (3) for various feedback strengths: $z=0.7$ (a), $z=2$ (b), $z=3$ (c). The solid lines represent the analytic prediction, the symbols represent numerical data obtained by increasing (filled circles) and decreasing (open circles) the coupling. The disorder is set to $\gamma=0.05$.

recall that in Fig. $1 N$ is changed through the simulations, while in Fig. $2 N$ is kept constant and very large to ensure the asymptotic limit $N \rightarrow \propto$ in Eq. (4). The comparison with numerical experiments such as those of Fig. 1 reveals that, at least for some values of the coupling with the resonator, the transition to the synchronous state is very similar in the JJ array and in the KM with $z>1$, the similarity being also discussed in Ref. [10] with a detailed discussion of the $z=2$ case. However the generalized KM has some features that are very interesting per se, and are the main objective of this work:

(1) For $z \leq 1$ the evolution from the incoherent value $r=0$ to the partially coherent state is continuous. Strictly speaking, there is no transition except for the special case $z=1$ where the expected second order phase transition is retrieved; for $z<1$ the order parameter is small but finite, and reaches zero only for $K=0$.

(2) The finite accuracy of the numerical simulations masks this difference between the special case $z=1$ and the $z<1$ cases. Presumably, in real systems a smooth, second orderlike transition would be observed also for systems best described by a negative feedback $z<1$.

(3) For $z>1$, as the parameter $K$ is increased past some critical value $K_{c}$, there is an onset of synchronization accompanied by a jump to a finite value of the order parameter ("first order phase transition").

(4) For $z>1$, as the parameter $K$ is decreased the numerical simulations show hysteretic behavior, with the transition back down to the $r=0$ state occurring at a lower coupling than $K_{c}$, even if the oscillators are activated and deactivated in the same order as has been done in Fig. 2.

The enforced coupling behavior modeled by $z>1$ is also in agreement with the results of findings of Ref. [15], where the order parameter $r$ increases for finite $N$, see Eq. (22) of Ref. [15] and with the experimental findings of a first order transition in some experiments in which the number of oscillators is varied $[8,9,14]$.

To analytically handle Eqs. (4) we case them in the form

$$
\dot{\vartheta}_{i}=\omega_{i}+K r^{z} \sin \left(\psi-\vartheta_{i}\right)
$$

Following the usual analysis [3,19], let us suppose that there exists a solution where $r$ and $\psi$ are time-independent, and that the synchronous state rotates at the peak frequency of the distribution $g$, i.e., in our reference system it is still $\left(\left\langle\omega_{i}\right\rangle=0\right)$. From Eq. (5) we get that the synchronized oscillators are just frozen with a fixed phase

$$
\omega_{i}=K r^{z} \sin \left(\vartheta_{i}^{*}\right) \Rightarrow \vartheta_{i}^{*}=\sin ^{-1}\left(\omega_{i} / K r^{z}\right) .
$$

Put another way, each oscillator is accommodated in a phase that depends upon the natural frequency of that specific oscillator $\left(\omega_{i}\right)$ and the global property of the synchronized state, the actual and unknown order parameter $r$. Another relevant consequence of Eq. (6) is that, depending on $K$, there will be a maximum frequency $\omega$ that can be synchronized. So one can divide the distribution $g(\omega)$ in two parts, a portion around zero of width $K r^{z}$ that participates in the synchronous motion (in this reference frame these phases are constant) and the outliers that rotate. Following Kuramoto we assume that an even $g(\omega)$ guarantees that the order parameter (2) evaluated for the outliers is zero (as many rotate clockwise as rotate counterclockwise), so the only nonvanishing contributions come from the central portion of the oscillators. To estimate $r$ for sufficiently many oscillators one approximates (3) with the integral 


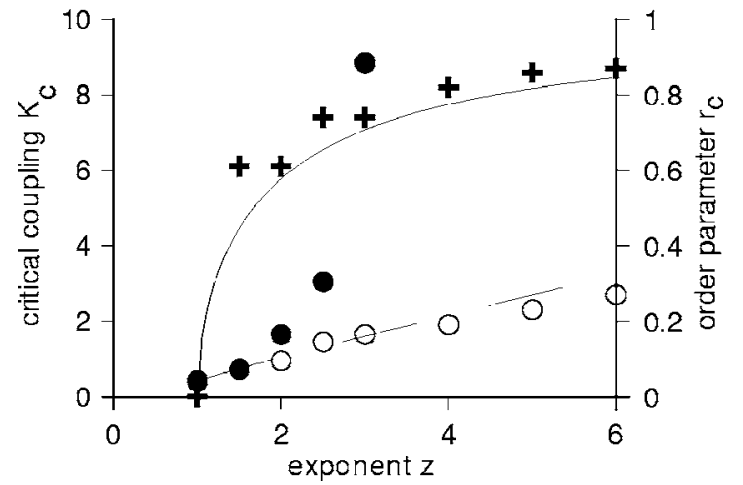

FIG. 3. Minimum value for the coupling parameter $K_{c}$ (dashed line, left axis) for the onset of synchronization and the corresponding value of the order parameter $r_{c}$ (solid line, right axis). Circles refer to the numerically determined critical coupling obtained by increasing (filled symbols) and decreasing (open circles) the coupling. Crosses refer to an estimate of the order parameter at the onset of synchronization. The disorder is set to $\gamma=0.05$.

$$
r e^{i \psi}=\frac{1}{N} \sum_{j=1}^{N} e^{i \vartheta_{j}} \cong \int_{-K r^{z}}^{K r^{z}} e^{i \vartheta} g(\omega) d \omega .
$$

For an even distribution $g(\omega)$ and $\omega$ given be Eq. (6), the self-consistency condition for the drifting oscillators requires that either $r=0$, or

$$
1=K r^{z-1} \int_{-\pi / 2}^{-\pi / 2} \cos ^{2}(\vartheta) g\left(K r^{z} \sin \vartheta\right) d \vartheta
$$

Assuming a Lorentzian distribution the condition (8) gives

$$
K=\left(2 \gamma r^{1-z}\right) /\left(1-r^{2}\right) .
$$

The critical value of the coupling $K_{c}$ can be retrieved observing that it corresponds to the minimum coupling, $K_{c}: \partial K / \partial r$ $=0$, so differentiating (9) one gets

$$
\begin{gathered}
r_{c}=\sqrt{z-1} / \sqrt{z+1}, \\
K_{c}=\gamma(z+1)[\sqrt{(z-1)} / \sqrt{(z+1)}]^{z-1} .
\end{gathered}
$$

Equations (9) and (10) correspond to the branch of coherent states, $r>0$, we have not been able to analytically treat the other branch. The predictions (10) are compared with numerical simulations in Fig. 3.

In conclusion, we have shown that a variation of the KM in which the coupling depends (via a new parameter $z$ ) on the fraction of synchronized oscillators has two interesting features. On one hand, it offers a phenomenological account for the transition to the synchronous state of globally coupled oscillators activated one by one. One the other hand since the parameter $z$ controls the degree of feedback provided by the other oscillators, one could speculate that the flexibility to tune the coupling offer the possibility to apply the generalized KM to wider class of coupled oscillators. The general model remains analytically tractable, and our analysis shows that the standard case $z=1$ is rather special. For $z<1$, which corresponds to a weakening of the coupling by increasing the order parameter $r$, no phase transition is predicted although the order parameter stays at a vanishingly small value. For $z>1$, which corresponds to a re-enforcing of the coupling by increasing the order parameter $r$, a first order phase transition is predicted.
[1] S. Strogatz, Sync: The Emerging Science of Spontaneous Order (Hyperion, New York, 2003).

[2] Y. Kuramoto, in International Symposium on Mathematical Problems in Theoretical Physics, edited by H. Araki, Lecture notes in Physics No. 30 (Springer, New York, 1975).

[3] J. A. Acebrón, L. L. Bonilla, C. J. Pérez Vicente, F. Ritort, and R. Spigler, Rev. Mod. Phys. 77, 137 (2005).

[4] K. Wiesenfeld, P. Colet, and S. H. Strogatz, Phys. Rev. Lett. 76, 404 (1996); Phys. Rev. E 57, 1563 (1998).

[5] M. Dhamala and K. Wiesenfeld, Phys. Lett. A 292, 269 (2002).

[6] R. Monaco, S. Pagano, and G. Costabile, Phys. Lett. A 131, 122 (1988).

[7] S. Pagano, R. Monaco, and G. Costabile, IEEE Trans. Magn. 25, 1080 (1989).

[8] P. Barbara, A. B. Cowthorne, S. V. Shitov, and C. J. Lobb, Phys. Rev. Lett. 82, 1963 (1999).

[9] A. N. Grib, P. Seidel, and J. Scherbel, Phys. Rev. B 65, 094508 (2002).
[10] G. Filatrella, N. F. Pedersen, and K. Wiesenfeld, Physica C 437-438, 65 (2006).

[11] H. Brusselbach, D. Cris Jones, M. S. Mangir, M. Minden, and J. L. Rogers Opt. Lett. 30, 1339 (2005).

[12] S. H. Strogatz, D. M. Abrams, A. McRobie, B. Eckhardt, and E. Ott, Nature (London) 438, 43 (2005).

[13] A. McRobie, G. Morgenthal, J. Lasenby, and M. Ringer, Proc. Inst. Civ. Eng., Struct. Build. 156(2), 71 (2003).

[14] A. McRobie (private communication).

[15] D. Pazó, Phys. Rev. E 72, 046211 (2005).

[16] G. Filatrella, N. F. Pedersen, and K. Wiesenfeld, Phys. Rev. E 61, 2513 (2000).

[17] H. A. Tanaka, A. J. Lichtenberg, and S. Oishi, Physica D 100, 279 (1997); Phys. Rev. Lett. 78, 2104 (1997).

[18] B. R. Trees, V. Saranathan, and D. Stroud, Phys. Rev. E 71, 016215 (2005).

[19] S. Strogatz, Lectures Notes in Biomathematics (Springer, 1993), Vol. 100, p. 122. 